\title{
Some Aspects of Plane Poiseuille Flow of a Polar Fluid through a Porous Layer with Brinkman Effects
}

\author{
M.T. KAMEL, M.H. HAMDAN \\ Department of Mathematical Sciences \\ University of New Brunswick \\ P.O. Box 5050, Saint John, New Brunswick, E2L 4L5 \\ CANADA
}

\begin{abstract}
Polar fluid through a porous structure is considered in an attempt to derive expressions for its effective viscosity under Poiseuille flow. Expressions for the velocity field and micro-rotation are obtained and compared with the corresponding expressions for flow in free space in order to illustrate the effects of the porous medium parameters on the traversing fluid. This work might be of applicability in the study of lubrication problems in configurations involving porous linings, and in the study of the effects of viscosity changes on oil well yield.
\end{abstract}

Key-Words:- Polar Fluid, Porous Media, Effective Viscosity

Received: April 20, 2021. Revised: September 30, 2021. Accepted: October 13, 2021. Published: October 23, 2021.

\section{Introduction}

Analysis of Poiseuille flow of Polar fluid has received considerable attention in the literature in order to better understand the behaviour of polar fluids and the effects of micro-rotation, and to provide for a measure of the effective viscosity. Interest in polar fluids stems from the various applications they enjoy, including modeling blood flow, analysis of lubrication mechanisms, geothermal energy studies and heat transfer, $[1,4$, $7,8,10]$, in addition to more recent applications of microchannels in the design of micro-fluidic devices, [9].

Increasing interest in polar fluid flow through and over porous surfaces is witnessed by the large number of recent studies which deal with deriving appropriate models of flow through porous structures, [11], analyzing flow in various porous settings, and modeling the boundary layer flow of a polar fluid over a porous surface or inside a porous medium. Polar fluid flow through porous structures may find applications in a number of areas, including the modeling of blood flow through synthetic tissues; in lubrication mechanisms with porous linings; and in geothermal heat transfer studies, to name only a few, $[5,6,7]$.

Less studied, however, is the Poiseuille flow of a polar fluid through a porous medium, which is the subject matter of this work. The objective is to derive the velocity and micro-rotation profiles and compare them with the results of Poiseuille flow through free-space in order to illustrate the effects of the porous medium parameters on the flow. In order to obtain an expression for the effective viscosity, we also obtain the velocity profile for the plane Poiseuille flow of a Newtonian fluid through a porous medium. This work was first presented in [7].

\section{Governing Equations}

Equations governing the unsteady flow of a polar fluid through a variable porosity medium are given by the following equation of continuity, linear momentum and angular momentum equation, [6]:

Conservation of Mass:

$\nabla \bullet \stackrel{\mathrm{r}}{q}=0$.

Linear Momentum Equation:

$\rho\left[\frac{\partial \stackrel{\mathrm{r}}{q}}{\partial t}+(\stackrel{\mathrm{r}}{q} \bullet \nabla)\left(\frac{\mathrm{r}}{\phi}\right)\right]=$

$-\varphi \nabla P+(\mu+\tau) \nabla^{2} \vec{q}+2 \tau \nabla \times \vec{G}-F(\mu+\tau) \vec{q}$

Angular Momentum Equation:

$\rho k^{2}\left[\frac{\partial \vec{G}}{\partial t}+(\vec{q} \bullet \nabla)\left(\frac{\vec{G}}{\varphi}\right)\right]=2 \tau \nabla \times \vec{q}+(\beta+\gamma) \nabla^{2} \vec{G}$

$+(\alpha+\beta-\gamma) \varphi \nabla\left(\frac{1}{\varphi} \nabla \bullet \vec{G}\right)-4 \tau \vec{G}$ 
where $\vec{G}$ is the micro-rotation vector, $\vec{q}$ is the specific discharge (that is, $\vec{q}=\varphi \vec{v}$, with $\vec{v}$ being the intrinsic averaged velocity), $P$ is the pressure, $t$ is the time variable, $\rho$ is the fluid density, $F$ is a porosity function that can be identified with $\frac{\varphi}{\eta}$ for slow flow, $\eta$ is the permeability, $\varphi$ is the porosity, $\mu$ is the fluid viscosity, $\tau$ is the rotational viscosity, $k$ is the radius of gyration, and $\alpha, \beta, \gamma$ are gradient viscosities.

If we take the rotational viscosity $\tau=0$ in (2), we recover the momentum equations governing flow of a Newtonian fluid through porous media (that is, the averaged form of Navier-Stokes equations, as given by Du Plessis and Masliyah [2,3]).

Now, consider the steady flow of a polar fluid through a porous medium bounded by parallel plates that extend in the $\mathrm{x}$-direction and located at $y=h$ and $y=-h$, as shown in Fig. 1.

$\mathrm{y}=\mathrm{h}$

Flow Direction

$y=-h$

Fig. 1: Representative Sketch

Assuming the flow is driven by a constant pressure gradient, equations (1), (2), and (3) reduce to the following when porosity $\varphi$ is constant:

$$
\begin{aligned}
& (\mu+\tau) u^{\prime \prime}+2 \tau g^{\prime}-F(\mu+\tau) u=\varphi \frac{d p}{d x} \\
& (\beta+\gamma) g^{\prime \prime}-4 \tau g-2 \tau u^{\prime}=0
\end{aligned}
$$

where $g$ is the component of the micro-rotation vector in the z-direction, and $u$ is the velocity component in the $\mathrm{x}$-direction, and prime notation denotes ordinary differentiation with respect to $y$.

Boundary conditions are the no-slip and no-spin on solid walls, namely

$$
g(-h)=g(h)=0 ; u(-h)=u(h)=0 \text {. }
$$

Equations (4) and (5) are rendered dimensionless with respect to $h$, and with respect to a characteristic velocity $U_{0}$ by defining:

$\left\{\begin{array}{l}u=U_{0} U ; y=h Y ; g=U_{0} G / h ; \eta=h^{2} \eta^{*} ; \\ F=\frac{\varphi}{h^{2} \eta^{*}}=\frac{f}{h^{2}} .\end{array}\right.$

In addition, we define:

$$
\left\{\begin{array}{l}
N^{2}=\frac{\tau}{\mu+\tau} ; L=\frac{L_{0}}{l}=2 h\left(\frac{\mu}{\beta+\gamma}\right)^{1 / 2} \\
A=\frac{\varphi h^{2} \frac{d p}{d x}}{U_{0}(\mu+\tau)}
\end{array}\right.
$$

Using (7) and (8) in (4) and (5) we obtain, respectively:

$$
\begin{aligned}
& G^{\prime \prime}-\left(\frac{N^{2} L^{2}}{1-N^{2}}\right) G-\frac{1}{2}\left(\frac{N^{2} L^{2}}{1-N^{2}}\right) U^{\prime}=0 \\
& U^{\prime \prime}+2 N^{2} G^{\prime}-f U=A
\end{aligned}
$$

Boundary conditions (6) take the form:

$$
G(-1)=G(1)=0 ; U(-1)=U(1)=0 \text {. }
$$

Now, differentiating (10), we obtain:

$U^{\prime \prime \prime}+2 N^{2} G^{\prime \prime}-f U^{\prime}=0$.

From (9) we obtain:

$U^{\prime}=2\left(\frac{1-N^{2}}{N^{2} L^{2}}\right) G^{\prime \prime}-2 G$.

Differentiating (13), twice, we obtain:

$U^{\prime \prime}=2\left(\frac{1-N^{2}}{N^{2} L^{2}}\right) G^{\prime \prime \prime}-2 G^{\prime}$

and

$U^{\prime \prime \prime}=2\left(\frac{1-N^{2}}{N^{2} L^{2}}\right) G^{i v}-2 G^{\prime \prime}$.

Using (13) and (15) in (12) we obtain: 
$G^{i v}-\left[f+N^{2} L^{2}\right] G^{\prime \prime}+f \frac{N^{2} L^{2}}{1-N^{2}} G=0$.

General solution to (16) is obtained as follows. Characteristic equation of (16) takes the form

$m^{4}-\left[f+N^{2} L^{2}\right] m^{2}+f \frac{N^{2} L^{2}}{1-N^{2}}=0$.

Roots of (17) are given by:

$$
m=\mp \Gamma_{1} ; \mp \Gamma_{2}
$$

where

$$
\begin{aligned}
\Gamma_{1} & =\sqrt{k_{1}+\sqrt{k_{1}{ }^{2}-k_{2}}} \\
\Gamma_{2} & =\sqrt{k_{1}-\sqrt{k_{1}^{2}-k_{2}}} \\
k_{1} & =\frac{f+N^{2} L^{2}}{2} \\
k_{2} & =f \frac{N^{2} L^{2}}{1-N^{2}} .
\end{aligned}
$$

General solution to (16) thus takes the form:

$$
G=\left\{\begin{array}{l}
c_{1} \cosh \Gamma_{1} Y+c_{2} \sinh \Gamma_{1} Y \\
+c_{3} \cosh \Gamma_{2} Y+c_{4} \sinh \Gamma_{2} Y
\end{array}\right.
$$

The following expression is then obtained for $U$, by inserting (23) in (13) and integrating once with respect to $Y$ :

$$
\begin{aligned}
& U=\left(c_{1} \sinh \Gamma_{1} Y+c_{2} \cosh \Gamma_{1} Y\right)\left\{2 \Gamma_{1} \frac{1-N^{2}}{N^{2} L^{2}}-\frac{2}{\Gamma_{1}}\right\} \\
& +\left(c_{3} \sinh \Gamma_{2} Y+c_{4} \cosh \Gamma_{2} Y\right)\left\{2 \Gamma_{2} \frac{1-N^{2}}{N^{2} L^{2}}-\frac{2}{\Gamma_{2}}\right\}+c_{5}
\end{aligned}
$$

Now, from (23) and (24) we obtain, respectively

$$
G^{\prime}=\left\{\begin{array}{l}
\Gamma_{1}\left(c_{1} \sinh \Gamma_{1} Y+c_{2} \cosh \Gamma_{1} Y\right) \\
+\Gamma_{2}\left(c_{3} \sinh \Gamma_{2} Y+c_{4} \cosh \Gamma_{2} Y\right)
\end{array}\right.
$$

$U^{\prime \prime}=2 \Gamma_{1}\left(c_{1} \sinh \Gamma_{1} Y+c_{2} \cosh \Gamma_{1} Y\right)\left\{\Gamma_{1}^{2} \frac{1-N^{2}}{N^{2} L^{2}}-1\right\}$

$$
+2 \Gamma_{2}\left(c_{3} \sinh \Gamma_{2} Y+c_{4} \cosh \Gamma_{2} Y\right)\left\{\Gamma_{2}^{2} \frac{1-N^{2}}{N^{2} L^{2}}-1\right\}
$$

Using (25), (26) in (10), we obtain:

$c_{5}=-\frac{A}{f}$.

Employing the boundary conditions (11) in (23) and (24), and making use of (27), we obtain:

$$
\begin{aligned}
& c_{1}=c_{3}=0 \\
& c_{2}=-\frac{A \sinh \Gamma_{2}}{f D} \\
& c_{4}=-\frac{A \sinh \Gamma_{1}}{f D}
\end{aligned}
$$

where

$$
D=2\left\{\begin{array}{l}
\left\{\Gamma_{2}\left(\frac{1-N^{2}}{N^{2} L^{2}}\right)-\frac{1}{\Gamma_{2}}\right\} \sinh \Gamma_{1} \cosh \Gamma_{2} \\
-\left\{\Gamma_{1}\left(\frac{1-N^{2}}{N^{2} L^{2}}\right)-\frac{1}{\Gamma_{1}}\right\} \sinh \Gamma_{2} \cosh \Gamma_{1}
\end{array} \mid\right.
$$

Using (29) and (30) in (23) and (24), we obtain:

$$
\begin{aligned}
& G=-\frac{A}{f D}\left\{\sinh \Gamma_{2} \sinh \Gamma_{1} Y+\sinh \Gamma_{1} \sinh \Gamma_{2} Y\right\} \text {. } \\
& U=-\frac{A}{f D}\left[\begin{array}{l}
D \\
+\left(2 \sinh \Gamma_{2} \cosh \Gamma_{1} Y\right)\left\{\Gamma_{1} \frac{1-N^{2}}{N^{2} L^{2}}-\frac{1}{\Gamma_{1}}\right\} \\
+\left(2 \sinh \Gamma_{1} \cosh \Gamma_{2} Y\right)\left\{\Gamma_{2} \frac{1-N^{2}}{N^{2} L^{2}}-\frac{1}{\Gamma_{2}}\right\}
\end{array}\right]
\end{aligned}
$$

Now, from equations (32) and (33) we obtain the following expressions for $g$ and $u$, respectively:

$$
g=\left(-\frac{h \frac{d p}{d x}}{2 \mu}\right) \frac{2 \eta^{*}\left(1-N^{2}\right)}{D}\left\{\begin{array}{l}
\sinh \Gamma_{2} \sinh \left(\Gamma_{1} y / h\right) \\
+\sinh \Gamma_{1} \sinh \left(\Gamma_{2} y / h\right)
\end{array}\right\}
$$


$u=\left(-\frac{h^{2} \frac{d p}{d x}}{2 \mu}\right) \frac{2 \eta^{*}\left(1-N^{2}\right)}{D}$

$\left[\begin{array}{c}D+2 \sinh \Gamma_{2} \cosh \left(\Gamma_{1} y / h\right)\left\{\Gamma_{1} \frac{1-N^{2}}{N^{2} L^{2}}-\frac{1}{\Gamma_{1}}\right\} \\ +2 \sinh \Gamma_{1} \cosh \left(\Gamma_{2} y / h\right)\left\{\Gamma_{2} \frac{1-N^{2}}{N^{2} L^{2}}-\frac{1}{\Gamma_{2}}\right\}\end{array}\right]$

By comparison, the corresponding solutions to Poiseuille flow of a polar fluid in the absence of a porous medium are given by [1]:

$g=\left(-\frac{h \frac{d p}{d x}}{2 \mu}\right)\left\{y / h-\frac{\sinh N L y / h}{\sinh N L}\right\}$

$u=\left(-\frac{h^{2} \frac{d p}{d x}}{2 \mu}\right)\left[\begin{array}{l}1-(y / h)^{2} \\ -\frac{2 N}{L}\left\{\frac{\cosh N L-\cosh N L y / h}{\sinh N L}\right\}\end{array}\right]$

\section{Volumetric Flow Rates and Effective Viscosities}

\section{(i) The case of polar fluid in free-space:}

In the case of plane Poiseuille flow of a polar fluid in free-space (that is, in the absence of the porous matrix), Cowin [1] obtained the following expression for the volumetric flow rate, $Q$ :

$Q=\int_{-h}^{h} u d y=-\frac{2 h^{3} \frac{d p}{d x}}{3 \mu}\left\{1+\frac{3}{L^{2}}-\frac{3 N}{L} \operatorname{coth} N L\right\}$

where $u$ is given by (37).

The base plane Poiseuille flow of a Newtonian fluid has the following volumetric flow rate:

$$
Q=\int_{-h}^{h} u d y=-\frac{2 h^{3} \frac{d p}{d x}}{3 \mu}
$$

Now, the effective viscosity, $\mu_{e f f}$, for plane Poiseuille flow can be defined in terms of $Q$. Thus, $\mu_{\text {eff }}$ is defined using (39) as:

$\mu_{e f f}=-\frac{2 h^{3} \frac{d p}{d x}}{3 Q}$

and the following $\mu_{\text {eff }}$ for polar fluid in Poiseuille flow is obtained by using (38) in (40):

$$
\mu_{e f f}=\frac{\mu}{\left\{1+\frac{3}{L^{2}}-\frac{3 N}{L} \operatorname{coth} N L\right\}}
$$

Now, (41) yields the following ratio of viscosities:

$$
\vartheta=\frac{\mu_{e f f}}{\mu}=\frac{1}{\left\{1+\frac{3}{L^{2}}-\frac{3 N}{L} \operatorname{coth} N L\right\}} .
$$

The ratio $\vartheta=\frac{\mu_{e f f}}{\mu}$ identifies the change in viscosity when a polar fluid flows through free-space relative to the viscosity of a Newtonian fluid in free-space. This reflects the effect of the fluid microstructure on the viscosity.

\section{(ii) The case of polar fluid in a porous medium:}

Volumetric flow rate for a polar fluid in the porous channel is obtained by using (35) in $Q=\int_{-h}^{h} u d y$. This yields

$Q=-\frac{2 h^{3} \frac{d p}{d x}}{3 \mu} \frac{3 \eta^{*}\left(1-N^{2}\right)}{D} Z$

where 
$Z=\left[D+2 \sinh \Gamma_{1} \sinh \Gamma_{2}\left\{\frac{2\left(1-N^{2}\right)}{N^{2} L^{2}}-\frac{1}{\Gamma_{1}^{2}}-\frac{1}{\Gamma^{2}{ }_{2}}\right\}\right]$

We can define the following three types of effective viscosities depending on the type of the base flow in plane Poiseuille flow in terms of volumetric flow rate, $Q$.

Type 1: When the base flow is that of a Newtonian fluid in free-space, $\vartheta_{1}=\mu_{\text {eff }} / \mu$ identifies the change in viscosity when a polar fluid flows through a porous medium relative to the viscosity of a Newtonian fluid. This reflects the combined effects of the porous medium and the fluid microstructure on the viscosity.

This is obtained by substituting $Q$ from (43) into (40). We thus have

$\vartheta_{1}=\frac{\mu_{e f f}}{\mu}=\frac{D}{3 \eta^{*}\left(1-N^{2}\right) Z}$

Type 2: When the base flow is that of a polar fluid in free-space, $\vartheta_{2}=\mu_{\text {eff }} / \mu$ identifies the change in viscosity when a polar fluid flows through a porous medium relative to the viscosity of a polar fluid in freespace. This reflects the effect of the porous medium on the viscosity.

This can be obtained by rewriting (38) as

$\mu_{\text {eff }}=-\frac{2 h^{3} \frac{d p}{d x}}{3 Q}\left\{1+\frac{3}{L^{2}}-\frac{3 N}{L} \operatorname{coth} N L\right\}$

and inserting $Q$ from (43) in (46). We thus have:

$\vartheta_{2}=\frac{\mu_{e f f}}{\mu}=\frac{D\left\{1+\frac{3}{L^{2}}-\frac{3 N}{L} \operatorname{coth} N L\right\}}{3 \eta^{*}\left(1-N^{2}\right) Z}$

Type 3: When the base flow is that of a Newtonian fluid in a porous medium, $\vartheta=\mu_{\text {eff }} / \mu$ identifies the change in viscosity when a polar fluid flows through a porous medium relative to the viscosity of a Newtonian fluid in the porous medium. This reflects the effect of the fluid microstructure on the viscosity.

Flow of a Newtonian fluid in the plane Poiseuille flow described in his work has the following velocity profile, obtained by solving (10) with $N=0$ : $u=-\frac{h^{2} \frac{d p}{d x}}{\mu} \eta^{*}\left\{1-\frac{\cosh y /\left(h \sqrt{\eta^{*}}\right)}{\cosh \left(1 / \sqrt{\eta^{*}}\right)}\right\}$

and the following volumetric flow rate:

$Q=-\frac{2 h^{3} \frac{d p}{d x}}{3 \mu} 3 \eta^{*}\left(1-\sqrt{\eta^{*}} \tanh \left(1 / \sqrt{\eta^{*}}\right)\right)$.

Writing (49) as

$\mu_{\text {eff }}=-\frac{2 h^{3} \frac{d p}{d x}}{3 Q} 3 \eta^{*}\left(1-\sqrt{\eta^{*}} \tanh \left(1 / \sqrt{\eta^{*}}\right)\right)$.

and inserting (43) in (50), we obtain

$\vartheta_{3}=\frac{\mu_{e f f}}{\mu}=\frac{\left(1-\sqrt{\eta^{*}} \tanh \left(1 / \sqrt{\eta^{*}}\right)\right) D}{\left(1-N^{2}\right) Z}$

The following relationships between the viscosity ratios derived above can easily be established:

$$
\begin{aligned}
& \vartheta_{2}=\vartheta_{1} / \vartheta \\
& \vartheta_{3}=3 \eta^{*} \vartheta_{1}\left(1-\sqrt{\eta^{*}} \tanh \left(1 / \sqrt{\eta^{*}}\right)\right)
\end{aligned}
$$

\section{Typical Parameter Values}

Numerical evaluation of viscosity ratios, velocity and micro-rotation profiles is predicated upon relationship between the polar fluid viscosity parameters, values of the porous medium parameters, roots of the characteristic equation (given by equation (17)), the driving pressure gradients, and the channel depth.

Material coefficients of the polar fluid are defined as:

$\tau$ : Relative Rotational Viscosity

$\alpha, \beta, \gamma:$ Viscosities of the gradient of total rotation

$\lambda, \mu$ : the usual viscosity coefficients,

and they satisfy the following conditions:

$$
\left\{\begin{array}{l}
\mu \geq 0 ; \tau \geq 0 ; \beta \geq 0 ; \gamma \geq 0 \\
3 \lambda+2 \mu \geq 0 \\
3 \alpha+2 \beta \geq 0
\end{array}\right.
$$

The material characteristic length, $l$, is defined in terms of the above viscosities by 
$l=\sqrt{\frac{\beta+\gamma}{\mu}}$

While the coupling number, $N$, is defined by

$$
N=\sqrt{\frac{\tau}{\mu+\tau}} ; 0 \leq N \leq 1 \text {. }
$$

The coupling number, $N$, is a property of the fluid, and is a measure of the degree to which a particle is constrained to rotate with the average angular velocity of the region in which it is embedded, [1].

Typical values for the porous medium parameter $f=\varphi / \eta^{*}$ are given in Table 1 , and some values of the roots of equation (17) are given in Table 2.

\begin{tabular}{|c|c|c|c|c|}
\hline$\eta^{*}$ & 0.1 & 0.5 & 0.9 & 0.99 \\
\hline $10^{-7}$ & $10^{6}$ & $5 \times 10^{6}$ & $9 \times 10^{6}$ & $9.9 \times 10^{6}$ \\
\hline $10^{-4}$ & $10^{3}$ & $5 \times 10^{3}$ & $9 \times 10^{3}$ & $9.9 \times 10^{3}$ \\
\hline $10^{-2}$ & 10 & 50 & 90 & 99 \\
\hline $10^{-1}$ & 1 & 5 & 9 & 9.9 \\
\hline 1 & $10^{-1}$ & 0.5 & 0.9 & 0.99 \\
\hline
\end{tabular}

Table 1: Values of $f=\varphi / \eta^{*}$

\begin{tabular}{|c|l|l|l|}
\hline$f$ & $L=2$ & $L=5$ & $L=5$ \\
& $N^{2}=0.1$ & $N^{2}=0.1$ & $N^{2}=0.9$ \\
\hline $10^{6}$ & $\Gamma_{1}=999.99$ & $\Gamma_{1}=999.99$ & $\Gamma_{1}=999.898$ \\
& $\Gamma_{2}=0.444$ & $\Gamma_{2}=2.778$ & $\Gamma_{2}=15.001$ \\
\hline $10^{3}$ & $\Gamma_{1}=31.622$ & $\Gamma_{1}=31.618$ & $\Gamma_{1}=26.494$ \\
& $\Gamma_{2}=0.667$ & $\Gamma_{2}=2.778$ & $\Gamma_{2}=17.903$ \\
\hline $10^{-1}$ & $\Gamma_{1}=0.619$ & $\Gamma_{1}=1.577$ & $\Gamma_{1}=4.643$ \\
& $\Gamma_{2}=0.340$ & $\Gamma_{2}=0.334$ & $\Gamma_{2}=1.021$ \\
\hline
\end{tabular}

Table 2: Roots of Characteristic Equation

\section{Conclusion}

In this work, we considered plane Poiseuille flow of a polar fluid through a porous structure and derived expressions for the velocity and micro-rotation. We derived expressions for a number of viscosity ratios that arise in the model used. Some typical values of the porous parameters and the fluid parameters have also been provided.

\section{References}

[1] S.C. Cowin, The theory of polar fluids, Adv. Appl. Mech., Vol. 14, 1974, pp. 279-347.

[2] J.P. Du Plessis and J.H. Masliyah, Mathematical Modeling of Flow through Consolidated Isotropic Porous Media, Transport in Porous Media, Vol. 3, 1988, pp. 145-161.

[3] J.P. Du Plessis and J.H. Masliyah, Flow through Isotropic Granular Porous Media, Transport in Porous Media, Vol. 6, 1991, pp. 207-221.

[4] A. C. Eringen, Theory of Micropolar Fluids, Journal of Mathematics and Mechanics, Vol.16, 1966, pp. 118.

[5] C.F. Chan Man Fong, M.N. Farah, M.H. Hamdan and M.T. Kamel, Polar Fluid Flow between Two Eccentric Cylinders: Inertial Effects, International Journal of Pure and Applied Mathematics, Vol. 23, No. 2, 2005, pp. 267-283.

[6] M.H. Hamdan and M.T. Kamel, Polar Fluid Flow through Variable-Porosity, Isotropic Porous Media Special Topics \& Reviews in Porous Media, Vol. 2 Issue 2, 2011, pp. 145-155.

[7] M.T. Kamel and M.H. Hamdan, Plane Poiseuille Flow of a Polar Fluid through Porous Media, 14th WSEAS MAMECTIS'12, Porto, Portugal, July $1-3,2012,6 \mathrm{pp}$.

[8] M.T. Kamel and M.H. Hamdan, Aspects of Thinfilm Polar Fluid Lubrication, Journal of Applied Mathematics and Computation, Vol. 80, No.1, 1996 , pp. 33-42.

[9] A. Kucaba-Pietal, Microchannels Flow Modelling with the Micropolar FluidTheory, Bulletin of the Polish Academy of Sciences: Technical Sciences, Vol. 52, No.3, 2004, pp. 209-214.

[10] G. Lukaszewicz, Micropolar Fluids: Theory and Applications, Birkhauser Boston, 1999.

[11] A. Raptis, Boundary Layer Flow of a Micropolar Fluid through a Porous Medium, Journal of Porous Media,Vol. 3, No. 1, 2000, pp. 95-97.

Creative Commons Attribution License 4.0 (Attribution 4.0 International, CC BY 4.0)

This article is published under the terms of the Creative Commons Attribution License 4.0

https://creativecommons.org/licenses/by/4.0/deed.en US 